\title{
General formalism for excitonic absorption edges in confined systems with arbitrary dimensionality
}

\author{
P. LEFEBVRE, P. CHRISTOL and H. MATHIEU
}

Groupe d'Etudes des Semiconducteurs, CNRS, Université Montpellier II, Case courrier 074, 34095 Montpellier cedex 5, France

\begin{abstract}
A metric space with a noninteger dimension $\alpha(1<\alpha)$ is used to describe bound and unbound states of strongly anisotropic Wannier-Mott excitons, such as those confined in semiconductor superlattices, quantum wells and quantum-well wires. Indeed, the relative motion of the electronhole pair which constitutes such excitons can never be considered strictly 1D, 2D or 3D. We calculate the optical absorption spectrum, near a Van Hove singularity, for any arbitrary value of the dimensionality $\alpha$. The whole absorption spectrum is obtained from a single compact equation, much simpler than the existing models. This model is an exact generalisation of the calculations performed, in the effective-mass approximation, for allowed transitions, by Elliott [Phys. Rev. 108, 1384 (1957)], in the 3-dimensional case, and by Shinada and Sugano [J. Phys. Soc. Japan 21, 1936 (1966)], for 2-dimensional media.
\end{abstract}

Metric spaces with noninteger dimensions are extensively used in mathematics, but have received quite a poor interest from physicists. However, the geometrical anisotropy of given interaction within a given medium can be faced $[1,2]$ quite conveniently by involving a fractional-dimensional interaction space. Then, a single noninteger parameter -the dimensionality $\alpha$ - is sufficient to describe the degree of anisotropy. For instance, Stillinger [1] gave a generalisation of Schrödinger wave mechanics to $\alpha$-dimensional ( $\alpha \mathrm{D})$ spaces, and Xing-Fei He [2] applied it to interband optical transitions and bound excitons in strongly anisotropic semiconductors. In this paper, we apply a fractional-dimensional model to the calculation of the absorption spectrum due to Wannier-Mott excitons in quantum wells, superlattices, or quantum wires. Our approach constitutes an accurate and handy method for simulating and optimizing the properties of future electro-optical and all-optical devices, based on confined excitons.

Absorption spectra due to bound and unbound states of 3D and 2D excitons have been respectively calculated by Elliott [3] and by Shinada and Sugano [4]. However, the internal relative motion of the electronhole pair in realistic heterostructures is never perfectly $1 D, 2 D$ or $3 D$, as one can easily judge from the energy of the ground (1s) state [2,5]. Altogether, the existing models need quite complicated numerical calculations : the energies and amplitudes of the discrete absorption peaks due to bound states are computed by variational models. For quasi-2D structures, the shape of the absorption due to the continuum of unbound states is estimated from the bidimensional model of Ref. 4. For quantum wires, the authors of Ref. 6 have calculated the Sommerfeld factor for the continuum in strictly ID Coulomb systems, for which they avoided the well-known divergence [7] by altering the shape of the potential.

In fact, the Coulomb interaction between electrons and holes near a Van Hove singularity can be described by a single dimensionality parameter $\alpha>1[2,8,9]$. In type-I systems, $\alpha$ can take any value between 2 and 3 for quantum wells and superlattices, and between 1 and 3 for quantum wires. In type-II systems, the 
Coulomb interaction might exhibit an effective dimension larger than 3 . The fractional-dimensional approach provides accurate and very simple expressions for the various physical characteristics, versus $\alpha$. Given an anisotropic system, there are several ways to determine $\alpha$. For instance, the energy of the ground state may be calculated by a variational method and injected into Eq. 2 (see below), directly yielding the value of $\alpha$. All the properties of the anisotropic exciton can be deduced from this single parameter. Other methods [8,9] allow to estimate a priori $\alpha$ from the physical characteristics of the structures. $\alpha$ is just a phenomenological parameter, from which we compute the entire optical density spectrum, near a critical point. We shall only give here the few equations necessary for this calculation.

Stillinger [1] has given the expressions for the Laplace operator, the angular-momentum $\mathrm{L}^{2}$ and the integration measure for a space of dimension $\alpha$. Taking the zero of energy as the band-gap at the singularity of interest, the Hamiltonian equation is restricted to the vicinity of this point by the standard envelope function formalism. Then the electron-hole relative motion is given by the so-called effective-mass equation (Wannier equation) which writes, in the $\alpha \mathrm{D}$ space [1,2] :

$\left[-\frac{\hbar^{2}}{2 \mu} \frac{1}{\mathrm{r}^{\alpha-1}} \frac{\partial}{\partial \mathrm{r}}\left(\mathrm{r}^{\alpha-1} \frac{\partial}{\partial \mathrm{r}}\right)-\frac{\mathrm{e}^{2}}{4 \pi \varepsilon \mathrm{r}}-\frac{1}{2 \mu \mathrm{r}^{2}} \frac{\hbar^{2}}{\sin ^{\alpha-2} \theta} \frac{\partial}{\partial \theta}\left(\sin ^{\alpha-2} \theta \frac{\partial}{\partial \theta}\right)\right] \psi(\mathrm{r}, \theta)=\mathrm{E} \cdot \psi(\mathrm{r}, \theta)$

where $\mu=\left(\mathrm{m}_{\mathrm{e}}^{-1}+\mathrm{m}_{\mathrm{h}}^{-1}\right)^{-1}$ is the reduced mass of the electron-hole pair, while $\mathrm{r}$ and $\theta$ represent the $\alpha \mathrm{D}$ polar pseudo-coordinates. The total envelope function is factorised into by two independent radial and an angular contributions, by writing $\psi(r, \theta)=\mathbf{R}(r) \Theta(\theta)$, where $\Theta(\theta)$ represents an eigen function of $\mathrm{L}^{2}$ with eigenvalue $\ell(\ell+\alpha-2)$. In turn, Eq. 1 is then divided into two uncoupled equations. The normalised solutions of the angular equation are proportional to Gegenbauer polynomials $C_{\ell}^{\alpha / 2-1}(\cos \theta)$, which are equivalent to Legendre polynomials for $\alpha=3$, and to Chebyshev polynomials for $\alpha=2[1,2]$. Concerning the radial part of the wave function, both cases of bound $(E<0)$ and unbound states $(E>0)$ must be considered. For bound states, the square-integrability of the wave function leads to quantized states determined by the integer quantum numbers $\mathrm{n}$ and $\ell$. Their energies are given by the following equation :

$$
E_{n}=-R y /[n+(\alpha-3) / 2]^{2}
$$

where Ry is the effective Rydberg energy of the $3 D$ exciton. The normalised eigenfunctions $R_{n}, \ell(r)$ are proportional to $r^{\ell} \cdot e^{-k_{n} r} \cdot L_{n-\ell-1}^{2 \ell+\alpha-2}\left(2 k_{n} r\right)[1,2]$, where $L_{i}^{j}(x)$ are Laguerre polynomials, $a_{B}$ is the effective Bohr radius of the 3D exciton, and the wave number $k_{n}=\left[(n+(\alpha-1) / 2) a_{B}\right]^{-1}$. For states in the continuum, there is no special condition for $r \rightarrow \infty$, so that there is no quantization of the energy into levels. Introducing the quantum number $\kappa$, such that $\kappa^{2}=2 \mu \mathrm{E} / \hbar^{2}$, and the dimensionless parameter $\gamma=(\mathrm{Ry} / \mathrm{E})^{1 / 2}$, the solutions are given by :

$\mathbf{R}_{\mathrm{K}, \ell}(\mathrm{r})=(2 \pi)^{(\alpha-2) / 2} \cdot \frac{\left|\Gamma\left(\ell+\mathrm{i} \gamma+\frac{(\alpha-1)}{2}\right)\right|}{\Gamma(2 \ell+\alpha-1)} \cdot \mathrm{e}^{\pi \gamma / 2}(2 \mathrm{kr})^{\ell} \cdot \mathrm{M}\left(2 \ell-\mathrm{i} \gamma+\frac{(\alpha-1)}{2}, 2 \ell+\alpha-1,-2 \mathrm{ikr}\right)(3)$.

$\Gamma(x)$ is Euler's Gamma function. $M(a, b, x)$ is Kummer's function, solution of the hypergeometric equation $x u^{\prime \prime}(x)+(b-x) u^{\prime}(x)-a u(x)=0$. The other terms are not determined by a normalisation condition, but rather by a choice of an asymptotic form for $R_{\kappa}, \ell(r)$, by a similar method as that explained in Ref. 4 .

The optical density of a structure is simply the logarithmic attenuation of the intensity of light across the whole sample. It involves the effective thickness $\mathrm{L}_{c}$ of the medium, i.e. its real thickness multiplied by a quality factor which is the electron-hole envelope-function overlap integral. $\mathbf{L}_{c}$ is the total length of the sample for bulk (3D) materials. In quantum wells, $\mathrm{L}_{c}$ is the well width for infinite potential wells, and a bit smaller than the well width for realistic structures with finite potential wells. In quantum wires, $\mathrm{L}_{c}$ is proportionnal to the square root of the effective cross sectional area of the wire. According to Elliott [7], the intensity of the absorption is proportional to the probability for finding the electron and the hole at the 
same place. Nonvanishing values only occur for $\ell=0$, i.e. for s-states (see above). If no broadening is assumed, the absorption by bound states is made of Dirac distributions centered on the energies of Eq. 2 , while the absorption above the band-gap is proportional to the product of the oscillator strength by the $\alpha \mathrm{D}$ conduction-to-valence joint density of states. The latter was given in Ref. 2. Detailed calculations will be published elsewhere [10]. We thus come directly to the result, i.e. the total optical density $O(\hbar \omega)$ for an $\alpha \mathrm{D}$ medium. We obtain a generalisation of Elliott's formula :

$\mathrm{O}(\hbar \omega)=\mathrm{O}_{0}\left\{\sum_{\mathrm{n}=1}^{\infty} \frac{\operatorname{Ry} \Gamma(\mathrm{n}+\alpha-2)}{(\mathrm{n}-1) !\left(\mathrm{n}+\frac{\alpha-3}{2}\right)^{\alpha+1}} \delta\left(\hbar \omega-\mathrm{E}_{\mathrm{n}}\right)+\frac{\left|\Gamma\left(\frac{\alpha-1}{2}+\mathrm{i} \gamma\right)\right|^{2} \mathrm{e}^{\pi \gamma} \gamma^{2-\alpha}}{2^{\alpha} \pi^{2-\alpha / 2} \Gamma(\alpha / 2)} \mathrm{Y}(\hbar \omega)\right\}$

$\mathrm{Y}(\mathrm{x})$ represents the Heavyside step function and

$\mathrm{O}_{0}=2^{2 \alpha-1} \omega\left|\mathrm{d}_{\mathrm{cv}}\right|^{2}[\Gamma(\alpha / 2)]^{2} \Gamma((\alpha-1) / 2) / \pi^{(\alpha-3) / 2} \mathrm{n}_{\mathrm{B}} \mathrm{c} \mathrm{Ry} \mathrm{a} \mathrm{B}_{\mathrm{B}}^{\alpha} \mathrm{L}_{\mathrm{c}}^{2-\alpha}[\Gamma(\alpha-1)]^{3}$

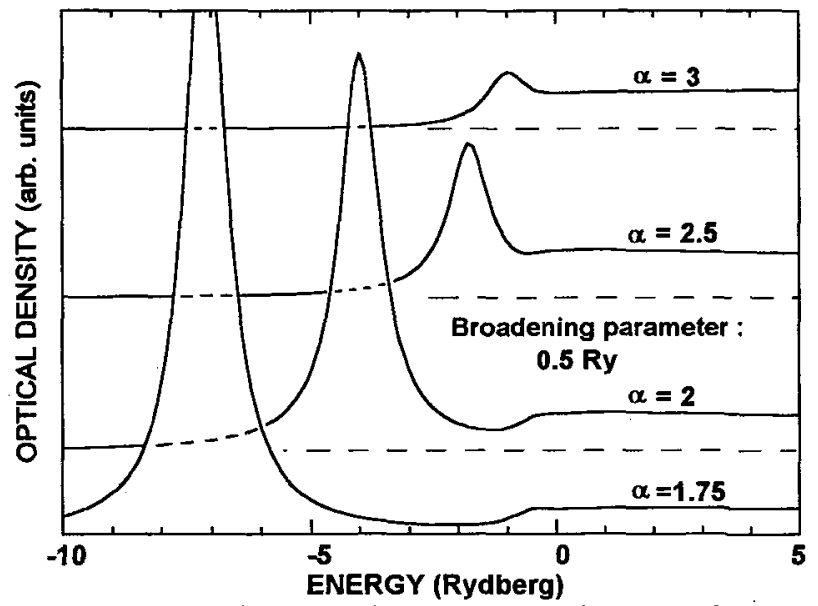

FIG. 1 : Calculated optical density spectra for several values of the effective dimensionality of the Coulomb interaction. These values correspond to the following ideal cases : $\alpha=3$ to a bulk material, $\alpha=2.5$ to a $1.4 a_{B}$-wide type I quantum well or a cylindrical quantum wire, with a diameter of $3.5 a_{B}$, $\alpha=2$ is either a vanishingly thin quantum well or a wire with a diameter of $1 a_{B}$, and $\alpha=1.75$ is reached by a wire of diameter $0.4 a_{B} . a_{B}$ is the effective Bohr radius of the $3 D$ exciton.

corresponds to the asymptotic case of an uncorrelated electron-hole pair. For integer values of $\alpha$, Eq. 4 yields well-established results : $\mathrm{O}_{3}(\gamma)=2 \pi \omega\left|\mathrm{d}_{\mathrm{cv}}\right|^{2} \mathrm{~L}_{\mathrm{c}} \mathrm{e}^{\pi \gamma} / \mathrm{n}_{\mathrm{B}} \mathrm{c} \mathrm{a}_{\mathrm{B}}^{3}$ Ry sinh $\pi \gamma$, which behaves like $\mathrm{E}^{1 / 2}$ at large $\mathrm{E}$, and $\mathrm{O}_{2}(\gamma)=2 \pi \omega\left|\mathrm{d}_{\mathrm{cv}}\right|^{2} \mathrm{e}^{\pi \gamma} / \mathrm{n}_{\mathrm{B}} \mathrm{c} \mathrm{a}_{\mathrm{B}}^{2} \mathrm{Ry} \cosh \pi \gamma$, which tends towards a constant. At the bandgap edge, i.e. for $\gamma \rightarrow \infty$, the continuous spectrum smoothly joins the quasi-continuous one.

In practical cases, for any dimension larger than 1 , the optical density of Eq. 4 is convoluted with a Lorentzian broadening function, to account for the finite lifetime of excitons. Fig. 1 displays examples of excitonic absorption onsets, for different dimensionalities corresponding respectively to the fundamental gaps in some ideal type I cases, detailed in the caption. The band-gap energy was taken as zero, to facilitate the comparison between all situations. We remark that spectra with the same general shape can corre-

where $\omega$ is the angular frequency of the incident light, $\mathrm{n}_{\mathrm{B}}$ the background refrac$\left|\mathrm{d}_{\mathrm{cv}}\right|^{2}=\left.|<\mathrm{c}| \mathrm{d}|\mathrm{v}\rangle\right|^{2}$ the conduction-to-valence squared matrix element of the electric dipole moment, at the considered critical point. The first term, at the righthand side of Eq. 4 gives the absorption by bound excitonic states. For $\mathrm{n} \rightarrow \infty$, the quantized levels tend to constitute a quasi-continuum, whose pseudo-density of states, varying like $[n+(\alpha-3) / 2]^{3}$ can be easily derived from Eq. 2. The fast decreasing of the oscillator strengths is then exactly compensated by this density, so that a finite value of the optical density is reached. In fact, this first term is the well-known factor which falls like $n^{-3}$ for $\alpha=3$ [3], and like $(n-1 / 2)^{-3}$ for $\alpha=2$ [4].

The second term, in the right-hand side of Eq. 4, accounts for the absorption by diffusion states, above the band-gap edge. At very high energies, i.e. for $\gamma \rightarrow$ 0 , this variation tends to behave like the $\alpha \mathrm{D}$ joint density of states [2], which

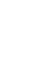


spond to either quantum wells or quantum wires, since these structures are characterized by the only dimensionality parameter. We also notice that our formalism could as well be used to treat quasi-1D absorption onsets of excitons in bulk materials under magnetic field [7].

Our model may also be suitable for treating type II systems, for which the binding energy can be smaller than $1 \mathrm{Ry}$. This indeed corresponds to values of $\alpha$ larger than 3, which have no reason to be discarded. In fact there are several clues in favor of this suggestion. First, the authors of Ref. 11 have evidenced a remarkable law stating that, for instance, the 3D 2p-state and the 5D 1s-state obey exactly the same radial equation. Both are therefore energetically equivalent. Now it is timeless that the limit case for perfect type II structures (infinite barrier between two semi-infinite media) is quite close to a $2 p_{z}$-like state, which strictly corresponds to a surface-donnor ground state [12]. Moreover, it is wellknown [5] that the interband absorption onset, in an ideal type II system, roughly varies like $E^{3 / 2}$. This is exactly the shape of the 5-dimensional joint-density of states of Ref. 2. Thus, as shown in Fig. 2, we suggest that the optical density spectra for type II excitons could be modelled by setting $\alpha>3$. Experiments on highquality samples should allow us to check the proposed lineshapes.

The model presented constitutes a powerful tool for simulating the so-called absorption spectrum of any kind of realistic microstructure [10], whatever its basic configuration (type I or type II). It

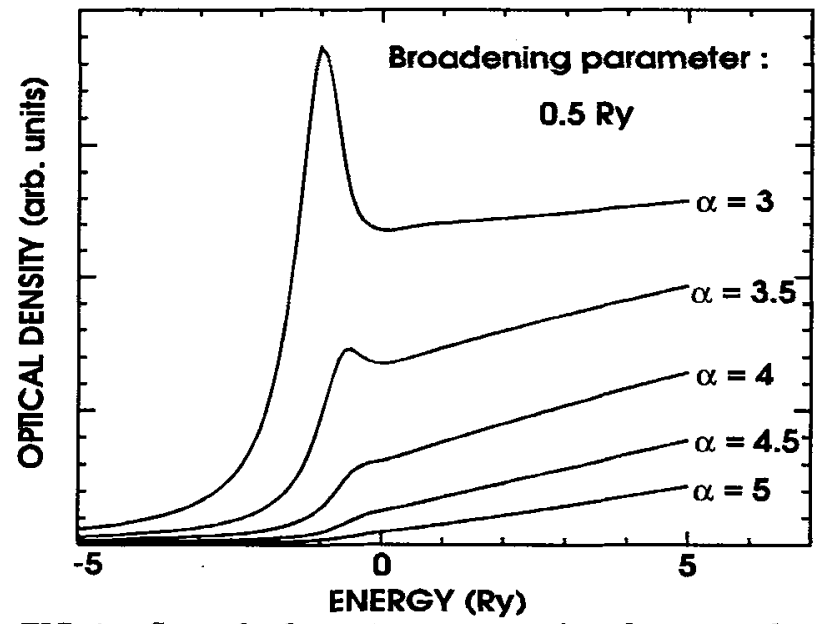

FIG. 2: Several absorption spectra for dimensionalities larger than 3. Such lineshapes should correspond to type II quantum wells or superlattices, or to any case where the usual Coulomb binding is weakened.

should also be particularly useful for simulating the effects of any kind of modulation on such spectrum. The only condition is that the modulated conduction and valence states and the corresponding binding energies of ground-state excitons are previously known.

To summarize, we may state that the concept of fractional-dimensional spaces is not a simple mathematical peculiarity. It can be applied to many physical systems, and may even exhibit many practical advantages, like in the case of anisotropic interactions. We have shown that using such a concept allows quite nice and compact formulations for the optical density spectra due to Wannier-Mott excitons in quantum wells, superlattices or quantum wires. This is of major interest for conceiving and optimizing future electro-optical or all-optical devices.

[1] Stillinger F.H., J. Math. Phys. 18, 1224 (1977)

[2] He X.F., Solid State Commun. 75, 111 (1990), also see : Phys. Rev. B 43, 2063, (1991).

[3] Elliott R.J., Phys. Rev. 108, 1384 (1957)

[4] Shinada M. and Sugano S., J. Phys. Soc. Japan 21, 1936 (1966)

[5] Bastard G., Wave mechanics applied to semiconductor heterostructures (Les Editions de Physique, Les Ulis, 1988). pp. 237-295

[6] Ogawa T. and Takagahara T., Phys. Rev. B 44, 8138 (1991)

[7] Elliott R.J. and Loudon J., J. Phys. Chem. Solids 8, 382 (1959); ibid. 15, 196 (1960)

[8] Mathieu H., Lefebvre P. and Christol Ph., Phys. Rev. B 46, 4092 (1992)

[9] Lefebvre P., Christol Ph. and Mathieu H., Phys. Rev. B 46, 13603 (1992)

[10]Lefebvre P., Christol Ph. and Mathieu H., unpublished

[11] Herrick D.R. and Stillinger F.H., Phys. Rev. A 11, 43 (1975)

[12] Levine J.D., Phys. Rev. 140, A586 (1965) 